\title{
COMPACT HOLOMORPHIC MAPPINGS ON BANACH SPACES AND THE APPROXIMATION PROPERTY
}

\author{
BY RICHARD M. ARON ${ }^{1}$ AND MARTIN SCHOTTENLOHER
}

Communicated by P. R. Halmos, May 2, 1974

1. Let $E$ be a complex Banach space. It is well known that $C(E ; C)$, the space of continuous scalar-valued functions on $E$ endowed with the compact-open topology, always has the approximation property, since there are continuous partitions of unity. However, for the space $H(E ; C)$ of holomorphic scalar-valued functions on $E$, the situation is more complicated. In $\S 2$ of this note, we describe this situation. Briefly, there is an exact analogy between the question of approximation by finite rank linear mappings on compact sets and the question of approximation by finite rank holomorphic mappings on compact sets.

In $\S 3$, we study the theory of compact holomorphic mappings between Banach spaces. The results of this section can be applied to characterize when $H(E ; C)$ has the approximation property, where $H(E ; C)$ is endowed with topologies other than the compact-open. This is of interest because, for many purposes, the compact-open topology is not the natural topology on $H(E ; C)$ (see for example [7]). In this note we are particularly concerned with the Nachbin "ported" topology $\tau_{\omega}$ on $H(E ; C)$. In $\S 4$, we characterize when $H(E ; C)$, endowed with $\tau_{\omega}$, has the approximation property. This result relates compact holomorphic mappings to the approximation property for $\left(H(E ; C), \tau_{\omega}\right)$ in a similar manner to the way compact linear mappings are related to the approximation property for $\left(E^{\prime}, \beta\right)$.

We shall follow [6] for notation and terminology for holomorphic mappings on Banach spaces and [5] for notation and terminology for the approximation property.

2. $E$ and $F$ will denote complex Banach spaces. $H(E ; F)$ denotes the space of holomorphic $F$-valued mappings on $E$; that is, mappings

AMS (MOS) subject classifications (1970). Primary 46B99, 46E10, 46E40, 46G99.

Key words and phrases. Approximation property, holomorphic mappings on Banach spaces, compact mappings.

1 The research of this author was supported by NSF Grant GP33117. 
$f: E \rightarrow F$ which have a Fre'chet derivative at every point of $E$. We shall denote by $\tau_{0}$ the compact-open topology on $H(E ; F)$, which is generated by the seminorms $f \rightarrow \sup \{\|f(x)\|: x \in K\}, f \in H(E ; F)$, where $K$ is a compact subset of $E$. We denote by $\tau_{\omega}$ the locally convex topology on $H(E ; F)$ generated by all seminorms ported by compact subsets of $E$. A seminorm $p$ on $H(E ; F)$ is said to be ported by the compact set $K \subset E$ if for every open set $V \supset K$, there is a constant $c(V)>0$ such that $p(f)$ $\leqslant c(V) \sup _{x \in V}\|f(x)\|$, for all $f \in H(E ; F)$.

THEOREM 1. The following statements are equivalent:

(i) $E$ has the approximation property.

(ii) $\left(H(E ; C), \tau_{0}\right)$ has the approximation property.

The implication (i) $\rightarrow$ (ii) is proved using the fact that a slight modification in Theorem 1 of [1] yields that $H(E ; C) \otimes G$ is dense in $(H(E ; G)$, $\tau_{0}$ ) for all locally convex spaces $G$. (ii) follows by an application of $[8$, Proposition 3.3] . (ii) $\rightarrow$ (i) follows by noting that $E_{c}^{\prime}$ is a complemented subspace of $\left(H(E ; C), \tau_{0}\right)$, where $E_{c}^{\prime}$ is the space $E^{\prime}$ endowed with the topology of uniform convergence on compact convex subsets of $E$.

Theorem 1 is the holomorphic analogue of the classical result that $E$ has the approximation property if and only if $E_{c}^{\prime}$ has the approximation property. As a result there are Banach spaces $E$ such that $\left(H(E, C), \tau_{0}\right)$ does not have the approximation property (see [4]).

Using the same methods, one can show that (i) and (ii) above are equivalent to the following condition:

(iii) The identity mapping id: $E \rightarrow E$ can be approximated uniformly on compact sets by holomorphic mappings from $E$ to $E$ of finite rank. That is, for each compact set $K \subset E$ and each $\epsilon>0$, there is a holomorphic mapping $f: E \rightarrow E$ with span $f(E)$ finite dimensional such that $\sup _{x \in K}\|f(x)-x\|<\epsilon$.

Thus, we get that approximation by finite rank holomorphic mappings is equivalent to approximation by finite rank linear mappings.

3. In this section, we introduce compact holomorphic mappings and describe some of their basic properties.

A mapping $f: E \rightarrow F$ is said to be compact if for each $x \in E$, there is a neighborhood $V_{x}$ of $x$ such that $f\left(V_{x}\right)$ is relatively compact in 
$F$. The space of compact holomorphic mappings from $E$ to $F$ is denoted $H_{K}(E ; F)$.

Note that when $E$ or $F$ is finite dimensional, $H_{K}(E ; F)=H(E ; F)$. It follows from the result below that compact holomorphic mappings share with compact linear mappings the property that it is sufficient to have "good behavior" at the origin.

Proposition 1. Let $f \in H(E ; F)$. Then $f \in H_{K}(E ; F)$ if and only if there is a 0-neighborhood $V_{0}$ in $E$ such that $f\left(V_{0}\right)$ is relatively compact in $F$.

However, unlike the linear case, it is not true that for any point $x \in E$, $f\left(V_{0}+x\right)$ is a relatively compact subset of $F$. In fact, if $E$ is infinite dimensional, there are functions $f \in H(E ; C)$ with the property that for each $\epsilon>0$ there is a point $x \in E,\|x\| \leqslant 1, \operatorname{such}$ that $\sup \{|f(y)|:\|y-x\|<\epsilon\}$ $=\infty$ (see [2]).

Another characterization of compact holomorphic mappings is the following, which shows that compact holomorphic mappings have a very "flat" image in a natural sense.

Proposition 2. Let $f \in H(E ; F)$. Then $f \in H_{K}(E ; F)$ if and only if there is a compact subset $L \subset F$ such that $f(E)$ is contained in the vector space spanned by $L$.

In addition, one can obtain characterizations of compact holomorphic mappings $f: E \rightarrow F$ in terms of the transpose mapping $f^{*}: H(F ; C) \rightarrow$ $H(E ; C)$, given by $f^{*}(g)=g \circ f$ for $g \in H(F ; C)$. The following is a useful example of such a characterization.

Proposition 3. Let $f \in H(E ; F)$. Then $f \in H_{K}(E ; F)$ if and only if the transpose mapping $f^{*}: F_{c}^{\prime} \rightarrow\left(H(E ; C), \tau_{\omega}\right)$ is continuous.

4. The results of $\S 3$ allow us to prove the following.

THEOREM 2. $\left(H(E ; C), \tau_{\omega}\right)$ has the approximation property if and only if the closure of $H(E ; C) \otimes F$ in $\left(H(E ; F), \tau_{\omega}\right)$ is $H_{K}(E ; F)$ for every Banach space $F$.

In order to prove Theorem 2 , one first shows that $\left(H_{K}(E ; F), \tau_{\omega}\right) \cong$ $L_{\epsilon}\left(F_{c}^{\prime},\left(H(E ; C), \tau_{\omega}\right)\right)$, which is the $\epsilon$-product of $\left(H(E ; C), \tau_{\omega}\right)$ and $F$ (see, for example, [9]), and then applies a recent result of Bierstedt and Meise [3].

Theorem 2 is the analogue of the well-known result for compact linear maps: $E^{\prime}$ with the norm topology has the approximation property if and 
only if for every Banach space $F$, the uniform closure of $E^{\prime} \otimes F$ in $L(E ; F)$ is the space of compact linear mappings from $E$ to $F$.

The problem of whether $\left(H(E ; C), \tau_{\omega}\right)$ has the approximation property can be reduced to a question about the approximation property for a sequence of Banach spaces.

Proposition 4. $\left(H(E ; C), \tau_{\omega}\right)$ has the approximation property if and only if for all $n \in N, P\left({ }^{n} E ; C\right)$ has the approximation property.

Here, $P\left({ }^{n} E ; C\right)$ is the space of continuous $n$-homogeneous polynomials from $E$ to $C$ with the sup norm. In fact, when $E=l_{1}$, then $\mathrm{P}\left({ }^{n} E ; C\right)$ is isomorphic to $l_{\infty}$ for $n \geqslant 1$, and so it follows that $\left(H\left(l_{1} ; C\right), \tau_{\omega}\right)$ has the approximation property. However, even for $E=l_{2}$, it is unknown whether $P\left({ }^{2} E ; C\right)$ has the approximation property; hence, it is unknown whether $\left(H(E ; C), \tau_{\omega}\right)$ has the approximation property.

Combining Proposition 4 and Theorem 1, we obtain the following

COROllary. If $\left(H(E ; C), \tau_{\omega}\right)$ has the approximation property, then $\left(H(E ; C), \tau_{0}\right)$ has the approximation property. The converse statement in general, is false.

Similar results to those given in Theorem 2 and Proposition 4 hold for two other useful topologies on $H(E ; C)$, namely for $\tau_{\infty}$ and $\tau_{b}$. Here, $\tau_{\infty}$ is the locally convex topology on $H(E ; F)$ of uniform convergence of a holomorphic mapping and each of its derivatives on compact sets, and $\tau_{b}$ is the bornological topology associated with $\tau_{0}$ (or equivalently with $\tau_{\omega}$ ) (see, for example, [1]).

Full details and complete proofs will appear in a later paper.

\section{BIBLIOGRAPHY}

1. R. Aron, Tensor products of holomorphic functions, Indag. Math. 35 (1973), $192-202$.

2. - Entire functions of unbounded type on a Banach space, Bol. Un. Mat. Ital. (4) 9 (1974), 28-31.

3. K.-D. Bierstedt and R. Meise, Einige Bemerkungen über die Approximationseigenschaft lokalkonvexer Räume, preprint, Kaiserslautern, 1973; Bemerkungen über die Approximationseigenschaft lokalkonvexer Funktionenräume, Math. Ann. 209 (1974), 99-107.

4. P. Enflo, A counterexample to the approximation property in Banach spaces, Acta Math. 130 (1973), 309-317.

5. A. Grothendieck, Produits tensoriels topologiques et espaces nucléaires, Mem. 
Amer, Math. Soc. No. 16 (1955). MR 17, 763.

6. L. Nachbin, Topology on spaces of holomorphic mappings, Ergebnisse der Mathematik und ihrer Grenzgebiete, Band 47, Springer-Verlag, New York, 1969. MR 40 \#7787.

7. - Recent developments in infinite dimensional holomorphy, Bull. Amer. Math. Soc. 79 (1973), 625-640.

8. M. Schottenloher, e-product and continuation of analytic mappings, Analyse Fonctionnelle et Applications, Hermann, Paris. (to appear)

9. L. Schwartz, Produits tensoriels topologiques d'espaces vectoriels topologiques, Séminaire 1953/54, Inst. Henri Poincaré.

DEPARTMENT OF MATHEMATICS, UNIVERSITY OF KENTUCKY, LEXINGTON, KENTUCKY 40506

Current address (R. M. Aron): 39 Trinity College, School of Mathematics, Dublin 2 , Ireland

Current address (M. Schottenloher): Mathematisches Institut der Universität München, D 8 München 2, Theresienstrasse 39, Federal Republic of Germany 